\title{
Study on the correlation between KCNJ11 gene polymorphism and metabolic syndrome in the elderly
}

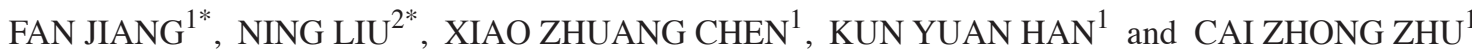 \\ Departments of ${ }^{1}$ Geratology and ${ }^{2}$ General Surgery, Hainan General Hospital, \\ Haikou, Hainan 570311, P.R. China
}

Received February 3, 2017; Accepted June 12, 2017

DOI: 10.3892/etm.2017.4714

\begin{abstract}
The aim of the study was to examine the correlation between KCNJ11 gene polymorphism and metabolic syndrome in elderly patients. From January 2014 to January 2015, 54 elderly patients with metabolic syndrome were enrolled in this study as the observation group. During the same period, 46 healthy elderly individuals were enrolled in this study as the control group. KCNJ11 gene polymorphism (rs28502) was analyzed using polymerase chain reaction-restriction fragment length polymorphism. The expression levels of mRNA in different genotypes were detected using FQ-PCR. ELISA was used to evaluate the KCNJ11 protein expression in different genotypes. KCNJ11 gene polymorphism and metabolic syndrome was studied by measuring the blood pressure levels in patients with different genotypes. Three genotypes of KCNJ11 gene in rs 28502 were CC, CT and TT. The CC, CT and TT genotype frequencies in healthy population were $8.5,9.2$ and $82.2 \%$, respectively, while the genotype frequencies in patients with metabolic syndrome were 42.4 , 49.8 and $7.8 \%$, respectively. There were significant differences between groups $(\mathrm{P} \leq 0.05)$. However, the genotype frequencies of $\mathrm{C} / \mathrm{T}$ in healthy individuals and metabolic syndrome patients were 35.3 and $38.3 \%$, respectively. There were no significant differences between groups $(\mathrm{P}>0.05)$. FQ-PCR results showed that the KCNJ11 mRNA expression levels in the control and observation groups had no significant differences $(\mathrm{P}>0.05)$. However, the results obtained from ELISA analysis revealed that KCNJ11 protein expression level in the observation group was significantly higher than that in the control group $(\mathrm{P}<0.05)$. In conclusion, KCNJ11 gene polymorphism is associated with metabolic syndrome in the elderly. Elderly patients with the $\mathrm{CC}$ and TT genotypes are more likely to develop metabolic syndrome.
\end{abstract}

Correspondence to: Dr Cai Zhong Zhu, Department of Geratology, Hainan General Hospital, 19 Xiuhua Road, Haikou, Hainan 570311, P.R. China

E-mail: caizhongzhu2017@163.com

*Contributed equally

Key words: KCNJ11, gene polymorphism, PCR-RFLP, metabolic syndrome in the elderly, genotype

\section{Introduction}

Metabolic syndrome (MS) is a polygenic inheritable disease caused by the combined effects of environmental factors and genetic factors (1). Obesity, hypertension and diabetes are the main clinical symptoms of metabolic syndrome (2). According to the clinical statistical data, MS has a notable correlation with a variety of diseases, for example, hypertension and heart disease are more common in elderly patients with metabolic syndrome. The deficiency of metabolic function leads to disorder in the body's energy supply, and negatively affects the immune system (3). This can create more important complications in elderly patients (4).

Few studies have been conducted on the pathogenesis of this condition in the elderly, and there is no effective medication for the treatment of metabolic syndrome in the elderly. The symptoms of metabolic syndrome are not significant at the early stage, which makes its diagnosis and treatment more difficult (5). It has been shown that KCNJ11, an 80-kDa protein, can participate in the secretion of insulin (6). A significant correlation between KCNJ11 gene and type 2 diabetes has been reported (7). KCNJ11 can inhibit the insulin release and increase the blood glucose level (8). At the same time, KCNJ11 can increase the level of lipids in bloodstream and also cause an increase in blood pressure. KCNJ11 gene contains one exon and one intron (9). The correlation between KCNJ11 gene polymorphism and metabolic syndrome in the elderly has not been studied extensively.

Thus, in the present study, we examined the correlation between KCNJ11 gene polymorphism and metabolic syndrome in the elderly. We provided theoretical and experimental basis for the diagnosis and treatment of metabolic syndrome in the elderly patients.

\section{Materials and methods}

General data. From January 2014 to January 2015, 54 elderly patients with metabolic syndrome who were admitted to the Hainan General Hospital (Haikou, China) were enrolled in this study as our observation group. There were 24 males and 30 females with an average age of 68.3.4 \pm 7.9 years. During the same period, 46 healthy elderly individuals were enrolled as the control group. There were 23 males and 23 females in the control group and their average age was $69.2 \pm 8.4$ years. 
Inclusion criteria were: i) Elderly patients with metabolic syndrome [according to the diagnostic criteria of metabolic syndrome in the elderly in 'Clinical Diagnosis and Treatment of Common Diseases in the Elderly' (10)]; ii) patients without other diseases; and iii) patients aged $>80$ years. Exclusion criteria were: i) Patients with non-metabolic syndrome; ii) patients with other related diseases, such as heart disease; and iii) patients $<60$ years of age.

Methods. The reagents, such as Ex Taq enzyme, dNTP, $6 \mathrm{X}$ buffer and genomic extraction kit were all purchased from Axygen (Union City, CA, USA). Agarose and GodView were both purchased from Solarbio Technology Co., Ltd. (Beijing, China), and other chemical reagents were purchased from Sangon Biotech (Shanghai, China).

Genomic extraction. The genomic DNA extraction kit was purchased from Axygen, and we closely followed the instructions provided by the manufacturer.

Polymerase chain reaction-restriction fragment length polymorphism (PCR-RFLP). Primers were synthesized by Sangon Biotech, and the primer sequences are shown in Table I. The PCR products were extracted and cloned.

KCNJ11 gene polymorphism. For the KCNJ11 polymorphism test, KCNJ11 gene was amplified via PCR, and PCR products were digested with SepI endonuclease (11).

Sequencing. Plasmids were extracted and submitted to Sangon Biotech for sequencing.

FQ-PCR

RNA extraction. Blood samples were collected from patients in both groups and used for RNA extraction.

$F Q-P C R$. In order to study the differences in the expression levels of KCNJ11 mRNA in different tissues, FQ-PCR was conducted. The primer sequences are shown in Table II.

ELISA. ELISA was conducted by closely following the protocol provided by $\mathrm{Li}$ (12).

Immunohistochemistry. Immunohistochemical assays were performed according to 'Current Protocols in Molecular Biology' (13).

Statistical analysis. The experimental data obtained were analyzed using SPSS 20.0 statistical software (SPSS, Chicago, IL, USA). The correlation between the gene and the differentiated thyroid carcinoma was studied using the ratio (OR) and 95\% confidence interval (CI). Unconditional logistic regression was used to calculate the OR value and the results were corrected by sex and other factors.

\section{Results}

Digestion of different KNSJ11 genotypes. The results obtained from enzyme digestion of PCR products revealed three bands with the sizes of 659,426 and $233 \mathrm{bp}$ for the elderly patients with metabolic syndrome after rs28502-site enzyme
Table I. KCNJ11 primer sequence.

\begin{tabular}{lc}
\hline Primer name & \multicolumn{1}{c}{ Sequence } \\
\hline KCNJ11 & F: GCCAGGGCCCCTCCTTCAA \\
KCNJ11 & R:TACCCTCAGACCCACGAGT
\end{tabular}

F, forward; R, reverse.

Table II. Primers of FQ-PCR.

\begin{tabular}{lc}
\hline Primer name & \multicolumn{1}{c}{ Sequence } \\
\hline qKCNJ11 & F: TGCTAGCTGATCGATCGATCGTCG \\
qKCNJ11 & R:CGTAGCTGATCGATGCTAGCTAGC \\
GAPDH & F: TGCTAGGCTAGGACGCTAGCTAC \\
GAPDH & R:CTGGGCTAGATCGACGAGAGCTC
\end{tabular}

F, forward; $R$, reverse.

Table III. Alleles between control and observation groups.

\begin{tabular}{lrrrrr}
\hline \multirow{5}{*}{ Groups } & Case (N) & $\mathrm{T}$ & $\mathrm{C}$ & $\chi^{2}$ & Pllele \\
\cline { 3 - 5 } & frequency (\%) & & \\
\hline Control & 108 & 48.4 & 43.3 & 14.04 & 0.026 \\
Observation & 92 & 45.3 & 40.3 & & \\
\hline
\end{tabular}

Table IV. Alleles between control and observation groups.

\begin{tabular}{lrrrrrr}
\hline & \multicolumn{5}{c}{$\begin{array}{c}\text { Genotype } \\
\text { frequency (\%) }\end{array}$} \\
\cline { 3 - 6 } Groups & Case (N) & CC & CT & TT & $\chi^{2}$ & P-value \\
\hline Control & 54 & 8.5 & 9.2 & 82.2 & 16.054 & 0.000 \\
Observation & 46 & 42.4 & 49.8 & 7.8 & & \\
\hline
\end{tabular}

digestion (Fig. 1). The results of sequencing showed that base mutation in rs28502 in the observation group was $\mathrm{T}$ or $\mathrm{C}$, namely KCNJ11 rs28502T/T and KCNJ11 rs28502 C/C with two bands with the sizes of 384 and $133 \mathrm{bp}$, while there was only 1 band at $517 \mathrm{bp}$ in the control group. The results of sequencing showed that its base sequence was KCNJ11 rs28502 T/C, and the sequencing is shown in Fig. 2. These results suggested that KCNJ11 gene in elderly patients with metabolic syndrome was mutated.

Alleles between control and observation groups. Results obtained from sequencing of KNSJ11 rs28502 in both groups revealed that the proportion of rs28502 allele $\mathrm{C}$ in the elderly patients with metabolic syndrome was $43.3 \%$ and that of $\mathrm{T}$ 


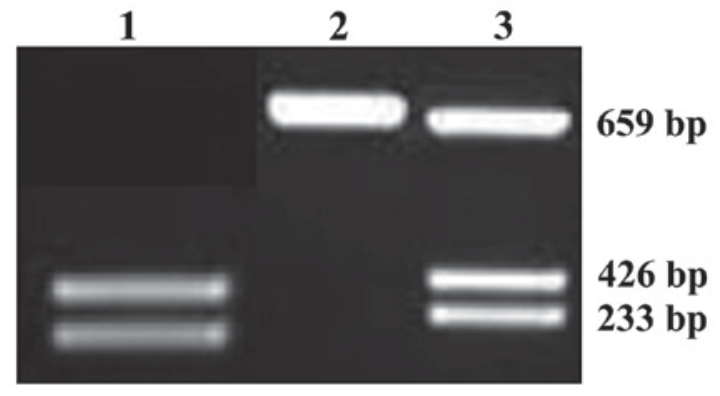

Figure 1. Results of enzyme digestion of different KNSJ11 genotypes. Lane 1 , sample of elderly patients with metabolic syndrome; lane 2 , sample of healthy individuals; and lane 3 , sample of elderly patients with metabolic syndrome.

A

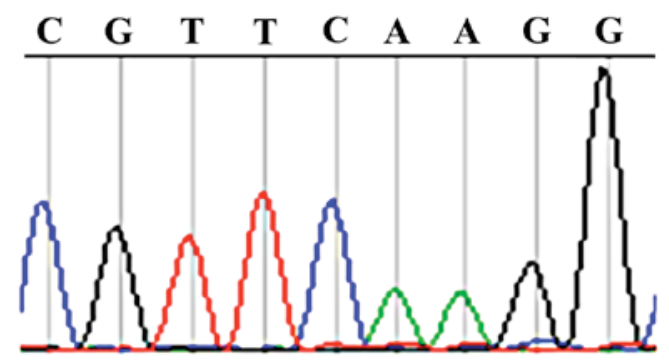

B

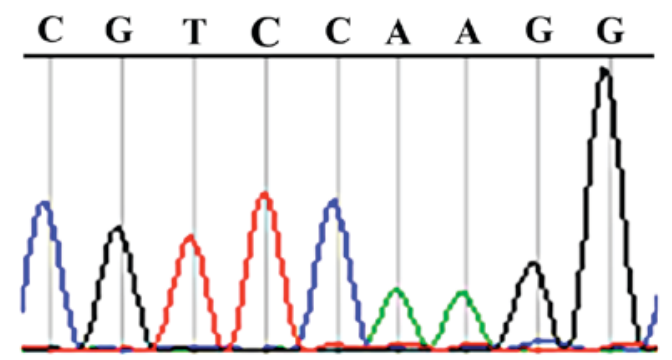

Figure 2. Results of gene sequencing. (A) Sequencing results of the healthy population. (B) Sequencing results of metabolic syndrome in the elderly.

was $48.4 \%$. We detected no significant differences between this proportion and the proportion of $\mathrm{C} / \mathrm{T}$ in the control group (40.3 and $45.3 \%$, respectively) $(\mathrm{P}>0.05)$ (Table III).

KNSJ11 genotypes between control and observation groups. KNSJ11 gene was amplified in both groups and sequencing results revealed polymorphism distribution in rs28502 base. Sequencing results showed that the proportions of CC, CT and TT in metabolic syndrome patients were $42.4,49.8$ and $7.8 \%$, respectively. These proportions in healthy subjetcs were 8.5, 9.2 and $82.2 \%$, respectively. These results suggested that the CT genotype was predominant for KNSJ11 in rs28502 site, while $\mathrm{CC}$ and $\mathrm{CT}$ genotypes were predominant for elderly patients with metabolic syndrome. There were significant differences in $\mathrm{CC}$ and TT genotypes between the groups $(\mathrm{P}<0.05)$ (Table IV).

Detection of MRNA expression in KNSJ11 gene between the control and observation groups. KNSJ11 mRNA expression levels in different samples are shown in Fig. 3. It can be seen

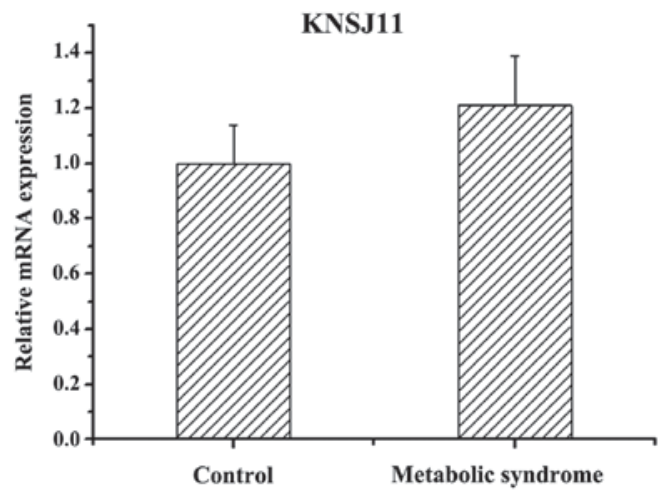

Figure 3. Detection of mRNA expression in KNSJ11 gene between the control and observation groups. $\mathrm{P}<0.05$.

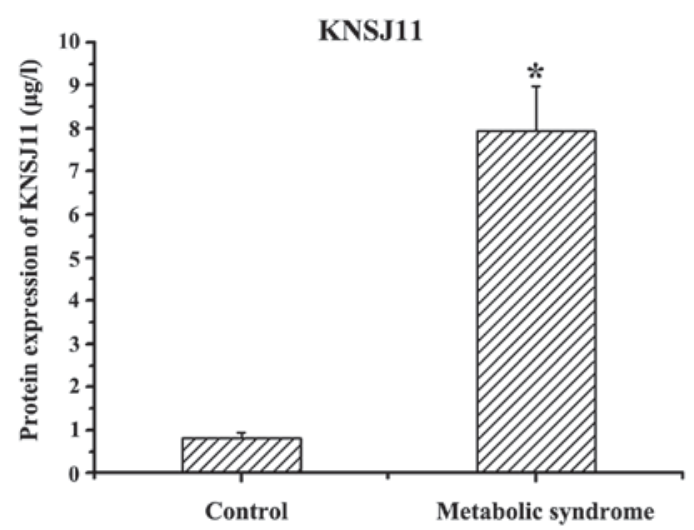

Figure 4. Detection of protein expression in KNSJ11 gene between the control and observation groups. ${ }^{*} \mathrm{P}<0.05$, significant difference between the two groups.

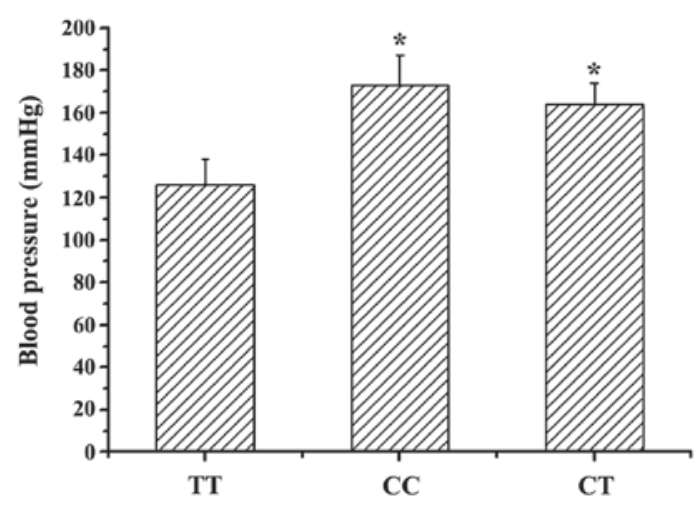

Figure 5. Detection of blood pressure (systolic pressure) in patients with different genotypes. ${ }^{*} \mathrm{P}<0.05$, significant difference between the two groups.

that compared with KNSJ11 mRNA expression level in the control group, the expression level was significantly higher in the metabolic syndrome. The difference was statistically significant $(\mathrm{P}<0.05)$.

Detection of protein expression in KNSJ11 gene between the control and observation groups. KNSJ11 protein expression level measured by ELISA is presented in Fig. 4. KNSJ11 protein expression level in the elderly patients with metabolic syndrome was significantly higher than that in the control 

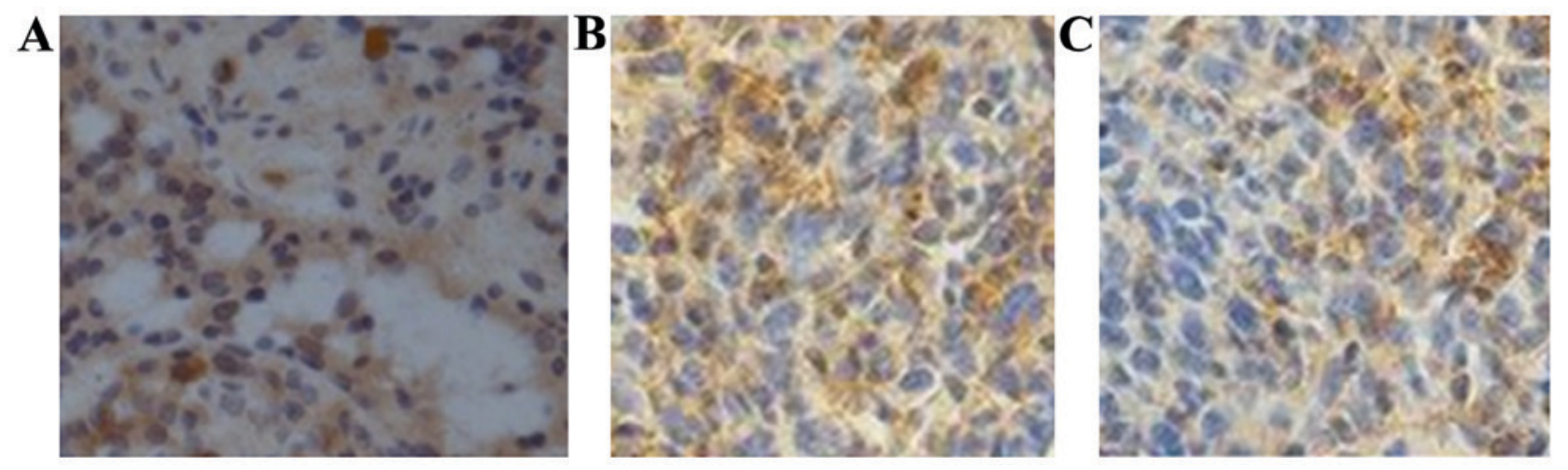

Figure 6. Immunohistochemical determination of patients in different genotypes. (A) Sample of healthy population, (B) sample of metabolic syndrome in the elderly with CT genotype, and (C) sample of metabolic syndrome in the elderly with CC genotype.

group $(7.94 \pm 1.03 \mu \mathrm{g} / 1$ vs. $0.83 \pm 0.12 \mu \mathrm{g} / 1$, respectively). The difference was statistically significant $(\mathrm{P}<0.05)$.

Detection of blood pressure (systolic pressure) in patients with different genotypes. To investigate the relationship between KNSJ11 genotype and metabolic syndrome in the elderly patients, blood pressure values in elderly patients with different genotypes were measured and compared. Results showed that the blood pressure levels in elderly patients with metabolic syndrome with $\mathrm{CC}$ and $\mathrm{CT}$ genotypes were significantly higher than that in TT genotype $(\mathrm{P}<0.05)$ (Fig. 5). The differences were statistically significant $(\mathrm{P}<0.05)$.

Immunohistochemical determination of patients in different genotypes. FQ-PCR results showed that there was no significant difference in the relative expression levels of mRNA in KNSJ11 gene in patients with different genotypes $(\mathrm{P}>0.05)$. Results of ELISA showed that there were significant differences in the KNSJ11 protein expression levels in patients with different genotypes $(\mathrm{P}<0.05)$. This indicated that different genotypes influenced the KNSJ11 protein expression level at the translational level. Immunohistochemical results showed that KNSJ11-positive cell count in elderly patients with metabolic syndrome was significantly higher than that in the healthy subjects (94.3 vs. $12.5 \%$, respectively) (Fig. 6). There were significant differences between the two groups $(\mathrm{P}<0.05)$, which was consistent with results obtained from ELISA.

\section{Discussion}

The increase in the size of the aging population in China has led to a significant increase in elderly-related diseases $(14,15)$. Metabolic syndrome in the elderly is a complex disease with cardiovascular and cerebrovascular diseases with obesity, hypertension and hyperglycemia as the main clinical symptoms (16). Metabolic syndrome in the elderly has a significant correlation with genetic information; however, the pathogenesis of metabolic syndrome in the elderly remains unknown. There is no efficient medication for metabolic syndrome in the elderly at present (17). Clinical data show that approximately $18.4 \%$ of elderly patients with metabolic syndrome suffer from varying degrees of fatty liver and abnormal blood lipid levels (18). Additional findings have shown that the fatty liver is usually accompanied with hypertension, diabetes and hyperlipidemia (19). In elderly patients with metabolic syndrome, the incidence of type 2 diabetes was significantly higher than that of patients with other diseases (20). Compared with healthy population, the KCNJ11 expression level in patients with metabolic syndrome is significantly different. KCNJ11 plays an important role in the process of $\mathrm{K}^{+}$transport. $\mathrm{K}^{+}$is important in maintaining the intracellular and extracellular osmotic pressure stability, maintaining the stability of cell membrane and promoting intracellular and intracellular exchange. It has been shown that there is a close relationship between KCNJ11 gene polymorphism and type 2 diabetes mellitus (21). KCNJ11 gene polymorphism can significantly increase the resistance of patients with type 2 diabetes to sulfonylurea drugs, and the polymorphism of KCNJ11 gene is also found in elderly patients with metabolic syndrome (21). KCNJ11 gene in patients with type 2 diabetes mellitus also shows a certain polymorphism; at the same time, we found that KCNJ11 gene in patients in different regions in China showed a certain level of polymorphism. We also found a correlation between the polymorphism and lifestyle habits. It has been shown that in the elderly suffering from metabolic syndrome, KCNJ11 gene polymorphism and type 2 diabetes are closely related. In the present study, we studied the correlation between KCNJ11 gene polymorphism and metabolic syndrome in elderly patients, and the results suggested that rs 28502 polymorphism of KCNJ11 gene had a significant correlation with the metabolic syndrome. The proportion of $\mathrm{CC}$ and $\mathrm{CT}$ genotypes in KCNJ11 gene in elderly patients with metabolic syndrome was significantly higher than that in healthy individuals. This result suggested that the elderly with $\mathrm{CT}$ and $\mathrm{CC}$ genotype are more likely to develop metabolic syndrome (22). FQ-PCR results revealed that KCNJ11 mRNA expression in patients with different genotypes had no significant difference. We also found that KCNJ11 protein expression levels in patients with $\mathrm{CC}$ and $\mathrm{CT}$ genotypes were significantly higher than those with TT genotype.

We concluded that KCNJ11 gene polymorphism was associated with metabolic syndrome in the elderly. we also concluded that elderly patients with the CC and TT genotypes are more likely to develop metabolic syndrome. 


\section{References}

1. Emanuela F, Grazia M, Marco de R, Maria Paola L, Giorgio F and Marco B: Inflammation as a link between obesity and metabolic syndrome. J Nutr Metab 2012: 476390, 2012.

2. Laguardia HA, Hamm LL and Chen J: The metabolic syndrome and risk of chronic kidney disease: Pathophysiology and intervention strategies. J Nutr Metab 2012: 652608, 2012.

3. Tao MH, Liu JW, LaMonte MJ, Liu J, Wang L, He Y, Li XY, Wang LN and Ye L: Different associations of apolipoprotein E polymorphism with metabolic syndrome by sex in an elderly Chinese population. Metabolism 60: 1488-1496, 2011.

4. Ong KL, Jiang CQ, Liu B, Jin YL, Tso AW, Tam S, Wong KS Tomlinson B, Cheung BMY, Lin JM, et al: Association of a genetic variant in the apolipoprotein A5 gene with the metabolic syndrome in Chinese. Clin Endocrinol (Oxf) 74: 206-213, 2011.

5. Jang Y, Chae JS, Kim OY, Park HJ, Kim JY, Paik JK, Lee SH and Lee JH: APOA 5-1131T>C genotype effects on apolipoprotein A5 and triglyceride levels in response to dietary intervention and regular exercise (DIRE) in hypertriglyceridemic subjects. Atherosclerosis 211: 512-519, 2010.

6. Akishita M, Fukai S, Hashimoto M, Kameyama Y, Nomura K, Nakamura T, Ogawa S, Iijima K, Eto M and Ouchi Y: Association of low testosterone with metabolic syndrome and its components in middle-aged Japanese men. Hypertens Res 33: 587-591, 2010.

7. Zhao $\mathrm{T}$ and Zhao J: Association of the apolipoprotein A5 gene $-1131 \mathrm{~T}>\mathrm{C}$ polymorphism with fasting blood lipids: A meta-analysis in 37859 subjects. BMC Med Genet 11: 120, 2010

8. Chaudhary R, Likidlilid A, Peerapatdit T, Tresukosol D, Srisuma S, Ratanamaneechat S and Sriratanasathavorn C: Apolipoprotein E gene polymorphism: Effects on plasma lipids and risk of type 2 diabetes and coronary artery disease. Cardiovasc Diabetol 11: 36, 2012.

9. Wang HL, Xu Q, Wang Z, Zhang YH, Si LY, Li XJ, Yang QH and $\mathrm{Xiao} \mathrm{H}$ : A potential regulatory single nucleotide polymorphism in the promoter of the Klotho gene may be associated with essential hypertension in the Chinese Han population. Clin Chim Acta 411: 386-390, 2010.

10. Majumdar V and Christopher R: Association of exonic variants of Klotho with metabolic syndrome in Asian Indians. Clin Chim Acta 412: 1116-1121, 2011.
11. Holstein A, Hahn M, Körner A, Stumvoll M and Kovacs P: TCF 7L2 and therapeuticresponse to sulfonylureas in patients with type 2 diabetes. BMC Med Genet 12: 30, 2011.

12. Li YY: The KCNJ11 E23K gene polymorphism and type 2 diabetes mellitus in the Chinese Han population: A meta-analysis of 6,109 subjects. Mol Biol Rep 40: 141-146, 2013.

13. Ausubel FM, Brent R, Kingston RE, Moore DD, Seidman JG, Smith JA and Struhl K (eds): Current Protocols in Molecular Biology. John Wiley \& Sons, Inc., Hoboken, NJ, 2011.

14. Zhang Q, Ramracheya R, Lahmann C, Tarasov A, Bengtsson M, Braha O, Braun M, Brereton M, Collins S, Galvanovskis J, et al: Role of KATP channels in glucose-regulated glucagon secretion and impaired counterregulation in type 2 diabetes. Cell Metab 18: 871-882, 2013.

15. Qin LJ, Lv Y and Huang QY: Meta-analysis of association of common variants in the KCNJ11-ABCC8 region with type 2 diabetes. Genet Mol Res 12: 2990-3002, 2013.

16. Abdelhamid I, Lasram K, Meiloud G, Ben Halim N, Kefi R, Samb A, Abdelhak S and Houmeida A: E23K variant in KCNJ11 gene is associated with susceptibility to type 2 diabetes in the Mauritanian population. Prim Care Diabetes 8: 171-175, 2014.

17. Martins L, Lourenço R, Maia AL, Maciel P, Monteiro MI, Pacheco L, Anselmo J, César R and Gomes MF: Transient neonatal diabetes due to a missense mutation (E227K) in the gene encoding the ATP-sensitive potassium channel (KCNJ11). Clin Case Rep 3: 781-785, 2015.

18. Qiu L, Na R, Xu R, Wang S, Sheng H, Wu W and Qu Y: Quantitative assessment of the effect of KCNJ11 gene polymorphism on the risk of type 2 diabetes. PLoS One 9: e93961, 2014.

19. Verderese JP and Younossi Z: Interaction of type 2 diabetes and nonalcoholic fatty liver disease. Expert Rev Gastroenterol Hepatol 7: 405-407, 2013.

20. Cho GJ, Shin JH, Yi KW, Park HT, Kim T, Hur JY and Kim SH: Serum calcium level is associated with metabolic syndrome in elderly women. Maturitas 68: 382-386, 2011.

21. Li C, Hsieh MC and Chang SJ: Metabolic syndrome, diabetes, and hyperuricemia. Curr Opin Rheumatol 25: 210-216, 2013.

22. Fujita T: Mineralocorticoid receptors, salt-sensitive hypertension, and metabolic syndrome. Hypertension 55: 813-818, 2010. 